Pacific Journal of Mathematic 


\section{A CONSTRUCTIVE PROOF OF SARD'S THEOREM}

\section{YUEN-KWOK CHAN}

The theorem of Sard asserts that if a mapping $F$ from a region in $R^{m}$ to $R^{p}$ is smooth enough, then the set of critical values of $F$ has measure zero in $R^{p}$. The purpose of this paper is to give a constructive proof of this theorem. By a constructive proof is meant one which has numerical content, as explained in E. Bishop's Foundations of Constructive Analysis. In particular, it is shown that in every open ball in $R^{p}$ one can compute a point which is not a critical value of $F$.

The proof is based on one given by Milnor, which is a modification of a proof of Pontryagin. These proofs, as well as all other known proofs, are nonconstructive, and it is not obvious that they can be constructivized. One difficulty lies in the fact that, given two real numbers $a$ and $b$, one cannot, in general, prove constructively that either $a \geqq b$ or $a<b$; one can only prove, for arbitrary $\varepsilon>0$, that either $a>b-\varepsilon$ or $a<b$. This fact forces, among other things, the consideration of 'nearly critical values' instead of critical values, and the derivation of a slightly more general result. Once a proper interpretation for "nearly critical values" has been found, Milnor's proof can be followed, replacing various nonconstructive arguments by constructive ones.

\section{Preliminaries.}

1.1. Suppose $f$ is a $C^{k}$ function (in other words, that $f$ has continuous partial derivatives of all orders not exceeding $k$ ) on a compact subset $U$ of $R^{m}$. If the positive constant $M$ is a bound for the absolute values of the partial derivatives of $f$ (of order at most $k$ and including $f$ itself) on $U$, then we say $M$ is a modulus of $k$-smoothness of $f$ on $U$. Given natural numbers $m$ and $k$, and a positive real number $\beta$, there are functions $\Phi_{k,+}, \Phi_{k, \times}, \Phi_{k, l}, \Phi_{k, s}$ from $(0, \infty) \times(0, \infty)$ to $(0, \infty)$ and a function $\Psi_{k}$ from $(0, \infty)$ to $(0, \infty)$ with the following properties:

(i) Suppose $f, g$ are $C^{k}$ functions on $U=\left[a_{1}, b_{1}\right] \times \cdots \times\left[a_{m}, b_{m}\right] \subset R^{m}$, and suppose $M_{f}$ and $M_{g}$ are respectively moduli of $k$-smoothness of $f$ and $g$ on $U$. Then the numbers $\Phi_{k,+}\left(M_{f}, M_{g}\right)$ and $\Phi_{k, \times}\left(M_{f}, M_{g}\right)$ are moduli of $k$-smoothness for $f+g$ and $f g$ respectively on $U$. If further $|g| \geqq \beta$, then $\Phi_{k, /}\left(M_{f}, M_{g}\right)$ is a modulus of $k$-smoothness for $f / g$ on $U$.

(ii) Suppose $f$ and $g$ are respectively $C^{k}$ functions on $U=$ $\left[a_{1}, b_{1}\right] \times \cdots \times\left[a_{m}, b_{m}\right]$ and on

$$
U^{\prime}=\left[a_{1}, b_{1}\right] \times \cdots \times\left[\widehat{a_{i}, b_{i}}\right] \times \cdots \times\left[a_{m}, b_{m}\right]
$$


(a hat signifies omission) with $g(y) \in\left[a_{i}, b_{i}\right]$ for each $y \in U^{\prime}$. If $M_{f}$ and $M_{g}$ are moduli of $k$-smoothness for $f$ and $g$ respectively, then the function $h$ defined on $U^{\prime}$ by $h(y)=f\left(x^{1}, \cdots, x^{i-1}, g(y), x^{i+1}, \cdots, x^{m}\right)$ (for each $y=\left(x^{1}, \cdots, \widehat{x^{i}}, \cdots, x^{m}\right)$ in $\left.U^{\prime}\right)$ will have $\Phi_{k, s}\left(M_{f}, M_{g}\right)$ as a modulus of $k$-smoothness.

(iii) Suppose $f$ is a $C^{k}$ function on $U=\left[a_{1}, b_{1}\right] \times \cdots \times\left[a_{m}, b_{m}\right]$ with $M_{f}$ as a modulus of $k$-smoothness and $\partial f / \partial x_{i} \geqq \beta$. If $g$ is a function from $U^{\prime}=\left[a_{1}, b_{1}\right] \times \cdots \times\left[\widehat{a_{i}, b_{i}}\right] \times \cdots \times\left[a_{m}, b_{m}\right]$ to $\left[a_{i}, b_{i}\right]$ such that $f\left(x^{1}, \cdots, x^{i-1}, g(y), x^{i+1}, \cdots, x^{m}\right)=0$ for every $y=\left(x^{1}, \cdots\right.$, $\left.\hat{x}^{i}, \cdots, x^{m}\right)$ in $U^{\prime}$, then $\Psi_{k}\left(M_{f}\right)$ is a modulus of $k$-smoothness for $g$ on $U^{\prime}$.

Existence of $\Phi_{k,+}, \Phi_{k, x}, \Phi_{k, l}$, and $\Phi_{k, s}$ are obvious. By taking their maximum we can assume that they all equal the same function $\Phi_{k}$. With notation as in (iii), the Implicit Function Theorem gives $\partial g / \partial x_{j}=$ $-\left(\partial f / \partial x_{j}\right) /\left(\partial f / \partial x_{i}\right)$, and similar formulas give higher order partial derivatives of $g$. From these the existence of $\Psi_{k}$ follows. Explicit forms of $\Phi_{k}$ and $\Psi_{k}$ can be found (e.g., $\left.\Phi_{k,+}\left(M, M^{\prime}\right)=M+M^{\prime}\right)$ but we shall not need them.

If $F=\left(F^{1}, \cdots, F^{p}\right)$ is a $C^{k}$ function from a compact set $U$ in $R^{m}$ to $R^{p}$, then a positive real number $M$ is said to be a modulus of $k$ smoothness of $F$ if it is at the same time moduli of $k$-smoothness of each of $F^{1}, \cdots, F^{p}$. The partial derivative $\partial F^{i} / \partial x_{j}$ will be written $F_{j}^{i}$.

1.2. If $m, p$ are natural numbers, $t=\min (m, p)$, and if $\left(a_{\jmath}^{i}\right)$ is a $p \times m$ matrix, then $D\left(\left(a_{j}^{i}\right)\right)$ will denote

$$
\max \left\{|\operatorname{det} S|: S \text { is a } t \times t \text { submatrix of }\left(a_{j}^{i}\right)\right\} \text {. }
$$

Suppose $F$ is a $C^{1}$ function from a compact subset $U$ of $R^{m}$ to $R^{p}$. Then $J_{F}$ will denote the function defined on $U$ by $J_{F}(x)=D\left(\left(F_{j}^{i}(x)\right)\right)$. It can easily be shown classically that $J_{F}(x)=0$ if and only if $F(x)$ is a critical value (i.e., if and only if the matrix $\left(F_{j}^{i}(x)\right)$ is of less than full rank.) Thus, roughly speaking, $F(x)$ is a nearly critical value if $J_{F}(x)$ is very small. Clearly there exists a function which assigns to every positive real number $M$ an operation $\omega(M):(0, \infty) \rightarrow(0, \infty)$ such that if $M$ is a modulus of 2 -smoothness of $F$ then $\omega(M)$ is a modulus of continuity of $J_{F}$.

1.3. To simplify the notation we introduce the symbol $\langle m, p, K$, $\Delta, F, M>$ to mean the statement:

$m, p$ are natural numbers with $p \geqq 1 ; K=\left[a_{1}, b_{1}\right] \times \cdots \times\left[a_{m}, b_{m}\right]$ where $0 \leqq a_{i} \leqq b_{i} \leqq 1$ for each $i \in\{1, \cdots, m\}, \Delta$ is a positive real number; $F$ is a $C^{k(m, p)}$ function from the closed neighborhood $U=$ $\left[a_{1}-\Delta, b_{1}+\Delta\right] \times \cdots \times\left[a_{m}-\Delta, b_{m}+\Delta\right]$ to $R^{p}$ with $M$ as a modulus of $k(m, p)$-smoothness on $U$, where $k(m, p)=2+2^{-1}(m-p)(m-p+1)$. 


\section{Sard's Theorem.}

THEOREM 2.1. Given natural numbers $m, p$ and positive real numbers $\Delta, M$, and $\varepsilon$, there exists a natural number $n=n(m, p, \Delta, M, \varepsilon)$ and a positive real number $\nu=\nu(m, p, \Delta, M, \varepsilon)$ such that if $\langle m, p, K$, $\Delta, F, M\rangle$ then the set $\left\{F(x): x \in K, J_{F}(x) \leqq \nu\right\}$ is contained in $n$ cubes in $R^{p}$ whose edges are equal and whose total volume is less than $\varepsilon$.

Proof of the theorem will be by induction, which is broken down into several lemmas.

LEMMA 2.2. Assume 2.1 is proved for a particular natural number $m-1(m \geqq 1)$. Then for every natural number $p \geqq 1$, and positive real numbers $\triangle, M, \varepsilon$, and $\beta$, there exist a natural number $n^{\prime}=n^{\prime}(m, p, \Delta, M, \varepsilon, \beta)$ and a positive real number $\nu^{\prime}=\nu^{\prime}(m, p, \Delta, M$, $\varepsilon, \beta)$ with the following properties: if $\langle m, p, K, \Delta, F, M\rangle$, and if for some $i \in\{1, \cdots, p\}$ and $j \in\{1, \cdots, m\}$ the partial derivative $F_{j}^{i}$ has absolute value always greater than $\beta$ on $U$, then $\left\{F(x): x \in K, J_{F}(x) \leqq \nu^{\prime}\right\}$ is contained in $n^{\prime}$ cubes in $R^{p}$ whose edges are equal and whose total volume is less than $\varepsilon$.

Proof. In case $p=1$ we can take $\nu^{\prime}=\beta / 2$ and $n^{\prime}=0$. For by assumption $J_{F} \geqq\left|F_{j}^{1}\right| \geqq \beta>\nu^{\prime}$ on $U$ and so our set $\left\{F(x): x \in K, J_{F}(x) \leqq \nu^{\prime}\right\}$ is void. Thus we can assume $p \geqq 2$. Without loss of generality we can also assume $M>1$ and $\beta<1 / 2$.

(i) Choose a natural number $q$ and then a positive real number $\varepsilon^{\prime}$ for which the following inequalities hold:

$$
q>6 \beta^{-1} \Delta^{-1} m M ; \varepsilon^{\prime}(q+1)^{m-1} 4^{p} M<\beta \varepsilon .
$$

Since 2.1 is proved for $m-1$, there exist a natural number $n_{0}=$ $n\left(m-1, p-1, q^{-1}, \Phi_{k}\left(M, \Psi_{k}(M)\right), \varepsilon^{\prime}\right)$ and a positive real number $\nu_{0}=$ $\nu\left(m-1, p-1, q^{-1}, \Phi_{k}\left(M, \Psi_{k}(M)\right), \varepsilon^{\prime}\right)$ having the properties as described in 2.1. Let $\varepsilon^{\prime \prime}$ be a positive real number and $d$ a natural number such that

$$
\begin{gathered}
\varepsilon^{\prime \prime}=\left(\varepsilon^{\prime} / n_{0}\right)^{1 /(p-1)}, \\
d^{-1}<(p-1)^{-1} M^{-1} \varepsilon^{\prime \prime},
\end{gathered}
$$

and

$$
d^{-1}<\omega(M)\left(2^{-1} \beta \nu_{0}\right)
$$

where $\omega$ was defined in 1.2 . Since $d \varepsilon^{\prime \prime}>1$ and $M \beta^{-1} d>1 \cdot 2 \cdot 1>1$, we can find natural numbers $\rho$ and $D$ such that 


$$
3 d \varepsilon^{\prime \prime}<\rho<4 d \varepsilon^{\prime \prime}
$$

and

$$
2 M \beta^{-1} d<D<4 M \beta^{-1} d
$$

Now let

$$
n^{\prime}=n_{0} \rho^{p-1} D(q+1)^{m-1}
$$

and let

$$
\nu^{\prime}=2^{-1} \beta \nu_{0} .
$$

We shall show $n^{\prime}$ and $\nu^{\prime}$ have the desired properties.

(ii) Thus suppose $\langle m, p, K, \Delta, F, M\rangle$ and (by relabelling) $\left|F_{1}^{1}\right| \geqq \beta$ on $U$. Without loss of generality assume $K=[0,1]^{m}$ and $F_{1} \geqq \beta$ on $U$. Let $\left\{\theta_{i}: i=1, \cdots,(q+1)^{m-1}\right\}$ be a family of cubes (in $[0,1]^{m-1}$ ) of edges $q^{-1}$ which covers $[0,1]^{m-1}$.

In (iii) and (iv) let $i$ be an arbitrary (but fixed) member of $\left\{1, \cdots,(q+1)^{m-1}\right\}$.

(iii) Since $D>2 M \beta^{-1} d$ and since $\left|F^{1}\right| \leqq M$ on $[0,1] \times \theta_{i}$ we can find $D$ points $x_{h}=\left(x_{h}^{1}, \cdots, x_{h}^{m}\right)(h=1, \cdots, D)$ in $[0,1] \times \theta_{i}$ such that $\left\{F^{1}\left(x_{h}\right)\right\}$ form a $\beta d^{-1}$-net for $F^{1}\left([0,1] \times \theta_{i}\right)$. Let $h \in\{1, \cdots, D\}$ be arbitrary. Let $U_{i}$ be the cube in $R^{m-1}$ with edge $3 q^{-1}$ and same center as $\theta_{i}$, the edges of $U_{i}$ being parallel to corresponding ones of $\theta_{i}$. Then for every $y=\left(x^{2}, \cdots, x^{m}\right)$ in $U_{i}$ we have

$$
\left|y^{j}-x_{h}^{j}\right| \leqq 3 q^{-1} \quad(j=2, \cdots, m) .
$$

If we let $c_{h}=F^{1}\left(x_{h}\right)$ it follows that

$$
\left|F^{1}\left(x_{h}^{2}, y\right)-c_{h}\right| \leqq 3 q^{-1} m M<\beta \Delta / 2 .
$$

Therefore, since $F_{1}^{1} \geqq \beta>0$, there exists $g^{h}(y) \in[-\Delta, 1+\Delta]$ such that

$$
F^{1}\left(g^{h}(y), y\right)-c_{h}=0 .
$$

By the Implicit Function Theorem and by definition of $\Psi_{k}$, the function $g^{h}$ is a $C^{k}$ function with $\Psi_{k}(M)$ as a $k$-smoothness modulus. $(k=$ $k(m, p)=k(m-1, p-1)$.) Now define a function

$$
G^{h}: U_{i} \longrightarrow R^{p-1}
$$

by

$$
G^{\dot{h}}(y)=\left(F^{2}\left(g^{h}(y), y\right), \cdots, F^{p}\left(g^{h}(y), y\right)\right) .
$$

Then $G^{h}$ is a $C^{k}$ function with $\Phi_{k}\left(M, \Psi_{k}(M)\right)$ as a $k$-smoothness modulus. Therefore, by the definitions of $n_{0}, \nu_{0}$, and $\varepsilon^{\prime \prime}$, the set

$$
B_{h}=\left\{G^{h}(y): y \in \theta_{i}, J_{G^{h}}(y) \leqq \nu_{0}\right\}
$$


is contained in $n_{0}$ cubes in $R^{p-1}$, each having edges $\varepsilon^{\prime \prime}$. Since $\rho>3 \varepsilon^{\prime \prime} d$, the $\varepsilon^{\prime \prime}$-neighborhood of $B_{h}$ is contained in $n_{0} \rho^{p-1}$ cubes of edges $d^{-1}$. Label these cubes $\eta_{h, 1}, \cdots, \eta_{h, n_{1}}$, where $n_{1}=n_{0} \rho^{p-1}$.

(iv) Suppose $x=\left(x^{1}, x^{2}, \cdots, x^{m}\right) \in[0,1] \times \theta_{i}$ is such that $J_{F}(x) \leqq \nu^{\prime}$. By definition of $\left\{x_{h}: h=1, \cdots, D\right\}$ there exists $h \in\{1, \cdots, D\}$ such that

$$
\left|F^{1}(x)-c_{h}\right|<\beta d^{-1}
$$

This implies, if we write $y=\left(x^{2}, \cdots, x^{m}\right)$, that

$$
\left|F^{1}\left(x^{1}, y\right)-F^{1}\left(g^{h}(y), y\right)\right|<\beta d^{-1} \text {. }
$$

Therefore, since $F_{1}^{1} \geqq \beta$, we have

$$
\left|g^{h}(y)-x^{1}\right|<d^{-1}
$$

We shall compute $J_{G^{h}}(y)$. For each $u \in\{2, \cdots, p\}$ and $v \in\{2, \cdots, m\}$, by definition of $G^{h}$ we have

$$
\begin{aligned}
\left(G^{h}\right)_{v}^{u}(y) & =F_{v}^{u}\left(g^{h}(y), y\right)+F_{1}^{u}\left(g^{h}(y), y\right) g_{v}^{h}(y) \\
& =\left\{\left[F_{1}^{1} F_{v}^{u}-F_{v}^{1} F_{1}^{u}\right] / F_{1}^{1}\right\}\left(g^{h}(y), y\right) .
\end{aligned}
$$

Let $t=\min (m-1, p-1)$ and let $S$ be any $t \times t$ submatrix of $\left(\left(G^{h}\right)_{v}^{u}(y)\right.$ ), say $S=\left(\left(G^{h}\right)_{v(s)}^{u(r)}(y)\right.$ ) (where $\left.r, s=1, \cdots, t\right)$. Then

$$
\begin{aligned}
& |\operatorname{det} S|=\left|\operatorname{det}\left(\left[F_{1}^{1} F_{v(s)}^{u(r)}-F_{v(s)}^{1} F_{1}^{u(r)}\right] / F_{1}^{1}\right)\left(g^{h}(y), y\right)\right|
\end{aligned}
$$

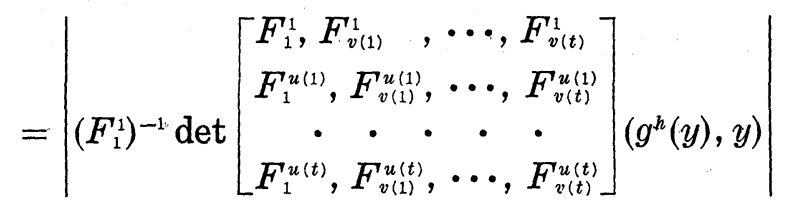

$$
\begin{aligned}
& \leqq \beta^{-1} J_{F}\left(g^{h}(y), y\right) \text {. }
\end{aligned}
$$

The second equality was obtained by an identity in the theory of determinants. Since $S$ was arbitrary we see

$$
J_{G^{h}}(y) \leqq \beta^{-1} J_{F}\left(g^{h}(y), y\right) \text {. }
$$

But $\left|g^{h}(y)-x^{1}\right|<d^{-1}<\omega(M)\left(2^{-1} \beta \nu_{0}\right)$ and $\omega(M)$ is by definition a continuity modulus for $J_{F}$. Hence

$$
\begin{aligned}
J_{F}\left(g^{h}(y), y\right) & \leqq J_{F}(x)+2^{-1} \beta \nu_{0} \\
& \leqq \nu^{\prime}+2^{-1} \beta \nu_{0}=\beta \nu_{0} .
\end{aligned}
$$

Combining, we have

$$
J_{G h}(y) \leqq \nu_{0}
$$

and so $G^{h}(y) \in B_{h}$. But 


$$
\begin{aligned}
\| & G^{h}(y)-\left(F^{2}(x), \cdots, F^{p}(x)\right) \| \\
=\|\left(F^{2}\left(g^{h}(y), y\right),\right. & \left.\cdots, F^{p}\left(g^{h}(y), y\right)\right)-\left(F^{2}\left(x^{1}, y\right), \cdots, F^{p}\left(x^{1}, y\right)\right) \\
& \leqq \\
& <(p-1) M\left|g^{h}(y)-x^{1}\right| \\
& (p-1) M d^{-1}<\varepsilon^{\prime \prime}
\end{aligned}
$$

Therefore $\left(F^{2}(x), \cdots, F^{p}(x)\right)$ is in the $\varepsilon^{\prime \prime}$-neighborhood of $B_{h}$ and so by (iii) is in one of the cubes $\eta_{h, 1}, \cdots, \eta_{h, n_{1}}$. On the other hand,

$$
\left|F^{1}(x)-c_{h}\right| \leqq \beta d^{-1} \leqq 2^{-1} d^{-1}
$$

Combining, we see

$$
\left\{\left[c_{h}-2^{-1} d^{-1}, c_{h}+2^{-1} d^{-1}\right] \times \eta_{h, j}: j=1, \cdots, n_{1} ; h=1, \cdots, D\right\}
$$

are cubes of edges $d^{-1}$, which together contain the set

$$
\left\{F(x): x \in[0,1] \times \theta_{i}, J_{F}(x) \leqq \nu^{\prime}\right\} .
$$

(v) Repeating the arguments in (iii) and (iv) for each $i$ in $\left\{1, \cdots,(q+1)^{m-1}\right\}$, we can enclose the set $\left\{F(x): x \in K, J_{F}(x) \leqq \nu^{\prime}\right\}$ in $n^{\prime}\left(=(q+1)^{m-1} n_{1} D\right)$ cubes of edges $d^{-1}$. It remains to check that $n^{\prime} d^{-p}<\varepsilon$. But this is immediate.

LeMMa 2.3. Assume 2.1 is proved for a particular natural number $m-1(m \geqq 1)$. Given a natural number $p \geqq 1$, positive real numbers $\Delta, M, \varepsilon$, and $\beta$, there exist a natural number $n^{\prime \prime}=n^{\prime \prime}(m, p, \Delta, M, \varepsilon, \beta)$ and a positive real number $\nu^{\prime \prime}=\nu^{\prime \prime}(m, p, \Delta, M, \varepsilon, \beta)$ with the following properties:

if

(i) $[m / p]>1$,

(ii) $\langle m, p, K, \Delta, F, M\rangle$,

(iii) for some $h \in\{1, \cdots,[m / p]-1\}$, some $(h+1)$ st partial derivative $F_{i_{1}, \cdots, i_{h+1}}^{j}$ has absolute value not less than $\beta$ on $U$, and

(iv) $B_{\nu}{ }^{\prime \prime}$ denotes the set $\left\{x \in K\right.$ : for all $r \in\{1, \cdots, p\}, h^{\prime} \in\{1, \cdots, h\}$, every $h^{\prime}-$ th partial derivative of $F^{r}$ has absolute value at $x$ not exceeding $\left.\nu^{\prime \prime}\right\}$, then the set $F\left(B_{\nu^{\prime \prime}}\right)$ is contained in $n^{\prime \prime}$ cubes in $R^{p}$ with equal edges and whose total volume is less than $\varepsilon$.

Proof. (i) Choose a natural number $q$ and then a positive real number $\varepsilon^{\prime}$ such that the following inequalities hold:

$$
\begin{gathered}
q>12 \beta^{-1} \Delta^{-1}(m-1) M ; \\
3^{p}(q+1)^{m-1} \varepsilon^{\prime}<\varepsilon .
\end{gathered}
$$

Let $k^{\prime}=k(m-1, p)$. Since we assume 2.1 is proved for $m-1$, there exist a natural number $n_{0}=n\left(m-1, p, q^{-1}, \Phi_{k^{\prime}}\left(M, \Psi_{k^{\prime}}(M)\right), \varepsilon^{\prime}\right)$ and a 
positive real number $\nu_{0}=\nu\left(m-1, p, q^{-1}, \Phi_{k^{\prime}}\left(M, \Psi_{k^{\prime}}(M)\right), \varepsilon^{\prime}\right)$ with properties as described in 2.1. Let $Q$ be a positive real number so small that whenever the entries of a $p \times(m-1)$ matrix $A$ are bounded in absolute value by $Q$ then $D(A) \leqq \nu_{0}$. Let

$$
\begin{gathered}
\varepsilon^{\prime \prime}=\left(\varepsilon^{\prime} / n_{0}\right)^{1 / p}, \\
n^{\prime \prime}=(q+1)^{m-1} n_{0},
\end{gathered}
$$

and let $\nu^{\prime \prime}$ be a positive real number so small that

$$
\begin{gathered}
\nu^{\prime \prime}\left(1+M \beta^{-1}\right)^{2}<Q, \\
\nu^{\prime \prime} M p^{1 / 2}<\beta \varepsilon^{\prime \prime},
\end{gathered}
$$

and

$$
\nu^{\prime \prime}<\beta \Delta / 8 \text {. }
$$

We shall show that $n^{\prime \prime}$ and $\nu^{\prime \prime}$ have the desired properties.

(ii) Therefore suppose $\langle m, p, K, \Delta, F, M\rangle$, suppose $[m / p]\rangle 1$, and suppose (after relabelling) $F_{i_{1}, \cdots i_{h}, 1}^{1} \geqq \beta$ on $U$. Without loss of generality assume $K=[0,1]^{m}$. Write $f=F_{i_{1}}^{1}, \ldots, i_{h}$. We can easily verify that

$$
\begin{aligned}
k(m, p)-h & \geqq k(m, p)-[m / p]+1 \\
& \geqq k(m-1, p)=k^{\prime} .
\end{aligned}
$$

Hence $f$ is a $C^{k^{\prime}}$ function with $M$ as a $k^{\prime}$-smoothness modulus. Let $\left\{\theta_{i}: i=1, \cdots,(q+1)^{m-1}\right\}$ be a family of cubes (in $[0,1]^{m-1}$ ) of edges $q^{-1}$ covering $[0,1]^{m-1}$. For each $i$ let

$$
m_{i}=\min \left\{|f(x)|: x \in[0,1] \times \theta_{i}\right\} .
$$

Partition $\left\{1, \cdots,(q+1)^{m-1}\right\}$ into subsets $P$ and $S$ such that

$$
\begin{aligned}
& m_{i}<2 \nu^{\prime \prime} \text { if } i \in P \text {, } \\
& m_{i}>\nu^{\prime \prime} \text { if } i \in S \text {. }
\end{aligned}
$$

For each $i \in P$ choose $x_{i}=\left(x_{i}^{1}, \cdots, x_{i}^{m}\right) \in[0,1] \times \theta_{i}$ such that $\left|f\left(x_{i}\right)\right|<2 \nu^{\prime \prime}$. Write $y_{i}=\left(x_{i}^{2}, \cdots, x_{i}^{m}\right)$.

(iii) Take any $i \in P$. Let $U_{i}$ be a cube in $R^{m-1}$ with same center as $\theta_{i}$ and edges $3 q^{-1}$, the edges being parallel to corresponding ones of $\theta_{i}$. Then for each $y=\left(y^{2}, \cdots, y^{m}\right) \in U_{i}$ we have

$$
\left|y^{j}-x_{i}^{j}\right| \leqq 3 q^{-1} \quad(j=2, \cdots, m) .
$$

Hence

$$
\begin{aligned}
\left|f\left(x_{i}^{1}, y\right)\right| & \leqq\left|f\left(x_{i}^{1}, y_{i}\right)\right|+3 q^{-1}(m-1) M \\
& <2 \nu^{\prime \prime}+\beta \Delta / 4<\beta \Delta / 2 .
\end{aligned}
$$


Therefore, since $f_{1} \geqq \beta$ on $U$, there is a point $g^{i}(y) \in[-\Delta, 1+\Delta]$ such that $f\left(g^{i}(y), y\right)=0$. By the Implicit Function Theorem and by the definition of $\Psi_{k^{\prime}}$, we see $g^{i}$ is a $C^{k^{\prime}}$ function with $\Psi_{k^{\prime}}(M)$ as a $k^{\prime}$ smoothness modulus. Now define a function

$$
G^{i}: U_{i} \longrightarrow R^{p}
$$

by

$$
G^{i}(y)=F\left(g^{i}(y), y\right)
$$

for each $y \in U_{i}$. Then $G^{i}$ is a $C^{k^{\prime}}$ function with $\Phi_{k^{\prime}}\left(M, \Psi_{k^{\prime}}(M)\right)$ as a $k^{\prime}$-smoothness modulus on $U_{i}$. Therefore, by definition of $n_{0}$ and $\nu_{0}$, there are cubes $\xi_{i, 1}, \cdots, \xi_{i, n_{0}}$ in $R^{p}$ of edges $\varepsilon^{\prime \prime}$ which cover $\left\{G^{i}(y)\right.$ : $y \in \theta_{i}, J_{G^{i}}(y) \leqq \nu_{0}$.

(iv) Now suppose $x=\left(x^{1}, \cdots, x^{m}\right) \in B_{\nu^{\prime \prime}}$. Write $y=\left(x^{2}, \cdots, x^{m}\right)$. Then $y \in \theta_{i}$ for some $i \in\left\{1, \cdots,(q+1)^{m-1}\right\}$. Since for this $i$ we have $m_{i} \leqq|f(x)| \leqq \nu^{\prime \prime}$, it follows that $i \in P$. Now $f_{1} \geqq \beta$ on $U$. Hence it. follows from

$$
\left|f\left(x^{1}, y\right)\right| \leqq \nu^{\prime \prime}
$$

and

$$
f\left(g^{i}(y), y\right)=0
$$

that

$$
\left|x^{1}-g^{i}(y)\right| \leqq \nu^{\prime \prime} \beta^{-1} .
$$

Therefore, for each $r \in\{1, \cdots, p\}$ and $s \in\{2, \cdots, m\}_{\text {L }}^{\mp}$ we have

$$
\begin{aligned}
\left|\left(G^{i}\right)_{s}^{r}(y)\right| & =\left|F_{s}^{r}\left(g^{i}(y), y\right)+F_{1}^{r}\left(g^{i}(y), y\right) g_{s}^{i}(y)\right| \\
& \leqq\left|F_{s}^{r}(x)\right|+\nu^{\prime \prime} \beta^{-1} M+\left|\left[F_{1}^{r} f_{s} / f_{1}\right]\left(g^{i}(y), y\right)\right| \\
& \leqq \nu^{\prime \prime}+\nu^{\prime \prime} \beta^{-1} M+\left|F_{1}^{r}(x)\right| \cdot\left|\left[f_{s} \mid f_{1}\right]\left(g^{i}(y), y\right)\right|+\nu^{\prime \prime} \beta^{-2} M^{2} \\
& \leqq \nu^{\prime \prime}\left(1+2 \beta^{-1} M+\beta^{-2} M^{2}\right) \\
& =\nu^{\prime \prime}\left(1+\beta^{-1} M\right)^{2} \leqq Q .
\end{aligned}
$$

Consequently, by definition of $Q$, we have

$$
J_{G^{i}}(y)=D\left(\left(G^{i}\right)_{s}^{r}(y)\right) \leqq \nu_{0} .
$$

Thus, by (iii), there is an $\alpha$ in $\left\{1, \cdots, n_{0}\right\}$ such that $G^{i}(y) \in \xi_{i, \alpha}$. Let $\xi_{i, \alpha}^{\prime}$ be the cube with the same center as $\xi_{i, \alpha}$, and with edges $3 \varepsilon^{\prime \prime}$, the edges being parallel to the corresponding ones of $\xi_{i, \alpha}$. Then, since

$$
\begin{aligned}
\left\|F(x)-G^{i}(y)\right\| & =\left\|F\left(x^{1}, y\right)-F\left(g^{i}(y), y\right)\right\| \\
& \leqq \nu^{\prime \prime} \beta^{-1} M p^{1 / 2}<\varepsilon^{\prime \prime},
\end{aligned}
$$

the point $F(x)$ is in $\xi_{i, \alpha}^{\prime}$. Summing up, we see that the $n^{\prime \prime}=(q+1)^{m-1} n_{0}$. cubes $\left\{\xi_{i, \alpha}: i=1, \cdots,(q+1)^{m-1} ; \alpha=1, \cdots, n_{0}\right\}$ (where we take $\xi_{i, \alpha}^{\prime}$ to 
be some arbitrary cube in $R^{p}$ with edges $3 \varepsilon^{\prime \prime}$ if $i \notin P$ ) form a cover for $F\left(B_{\nu^{\prime \prime}}\right)$. It remains to show $n^{\prime \prime}\left(3 \varepsilon^{\prime \prime}\right)^{p}<\varepsilon$. But this is immediate.

2.4. Proof of 2.1. The theorem is clearly true for $m=0$. Assume it is proved for some particular number $m-1(m \geqq 1)$.

(i) We are given a natural number $p \geqq 1$ and positive real numbers $\Delta, M$, and $\varepsilon$. Let

$$
\begin{gathered}
\lambda=[m / p], \\
\varepsilon^{\prime}=2^{-p-2}(\lambda+1)^{-1} \varepsilon .
\end{gathered}
$$

Let $q_{\lambda}$ be a natural number so large that

$$
q_{\lambda}^{\lambda+1-(m / p)}\left(\varepsilon^{\prime}\right)^{1 / p} \geqq 2^{(m / p)+2} M((\lambda+1) !)^{-1} m^{\lambda+1}
$$

and let $\delta_{\lambda}$ be a positive real number so small that

$$
\delta_{\lambda} \sum_{h=1}^{\lambda} m^{h} / h ! \leqq 2^{-(m / p)-2} q_{\lambda}^{-m / p}\left(\varepsilon^{\prime}\right)^{1 / p} .
$$

Inductively define $q_{h}, \varepsilon_{h}, \delta_{h}$, and $n_{h}(h=\lambda-1, \lambda-2, \cdots, 0)$ in the following way: For each natural number $h$ such that $0 \leqq h<\lambda$, let $q_{h}$ be a natural number such that

$$
m^{-1 / 2} q_{h}>\Delta^{-1} \text { and } q_{h}>24 m M \delta_{h+1}^{-1},
$$

and let $\varepsilon_{h}$ be a positive real number so small that

$$
\left(q_{h+1}\right)^{m} \varepsilon_{h}<\varepsilon^{\prime} .
$$

If $0<h<\lambda$ let $n_{h}=n^{\prime \prime}\left(m, p, q_{h}^{-1}, M, \varepsilon_{h}, 2^{-3} \delta_{h+1}\right)$ and $\delta_{h}=\nu^{\prime \prime}\left(m, p, q_{h}^{-1}\right.$, $M, \varepsilon_{h}, 2^{-3} \delta_{h+1}$ ) where $n^{\prime \prime}$ and $\nu^{\prime \prime}$ are as given in 2.3. If $0=h<\lambda$ let $n_{h}=n^{\prime}\left(m, p, q_{h}^{-1}, M, \varepsilon_{h}, 2^{-3} \delta_{h+1}\right) \quad$ and $\quad \delta_{h}=\nu^{\prime}\left(m, p, q_{h}^{-1}, M, \varepsilon_{h}, 2^{-3} \delta_{h+1}\right)$ where $n^{\prime}$ and $\nu^{\prime}$ are as given in 2.2. Without loss of generality we may take $\delta_{h}<\delta_{h+1}$. Finally, it is obvious that we can find a natural number $n$ with the following properties:

If we have $\left(q_{\lambda}+1\right)^{m}$ cubes in $R^{p}$ of equal edges and of total volume not exceeding $\varepsilon^{\prime}$, and if for each natural number $h$ with $0 \leqq h<\lambda$ we are given $\left(q_{h}+1\right)^{m} n_{h}$ cubes in $R^{p}$ of equal edges and of total volume not exceeding $\varepsilon^{\prime}$, then we can find $n$ cubes in $R^{p}$ of equal edges and of total volume not exceeding $2^{p+1}(\lambda+1) \varepsilon^{\prime}$, which cover all the given cubes.

Take such an $n$. We shall show $n$ and $\nu=\delta_{0}$ have the desired properties.

(ii) Thus suppose $\langle m, p, K, \Delta, F, M\rangle$. Without loss of generality we may assume $K=[0,1]^{m}$. If $\lambda=0$ let $C_{0}=K$. If $\lambda>0$ let

$$
C_{2}=\{x \in K \text { : all partial derivatives of } F \text { of order } h
$$

$(1 \leqq h \leqq \lambda)$ have absolute value at $x$ bounded by $\left.\delta_{\lambda}\right\}$, 
let for each $h(0<h<\lambda)$

$C_{h}=\left\{x \in K\right.$ : all partial derivatives of $F$ of order $h^{\prime}$

$\left(1 \leqq h^{\prime} \leqq h\right)$ have absolute values at $x$

bounded by $\delta_{h}$, but some $(h+1)$ st partial derivative

has absolute value at $x$ greater than $\left.2^{-1} \delta_{h+1}\right\}$,

and let

$C_{0}=\{x \in K$ : some first partial derivative of $F$ has absolute value at $x$ greater than $2^{-1} \delta_{1}$, but $J_{F}(x) \leqq \nu$.

Then, since $\delta_{h}<\delta_{h+1}(h=0, \cdots, \lambda-1)$, the set $\left\{x \in K: J_{F}(x) \leqq \nu\right\}$ is contained in $C_{0} \cup C_{1} \cup \cdots \cup C_{\lambda}$.

(iii) Let $\left\{\theta_{i}: i=1, \cdots,\left(q_{\lambda}+1\right)^{m}\right\}$ be a family of cubes in $[0,1]^{m}$ of edges $q_{\lambda}^{-1}$, which covers $[0,1]^{m}$. For each $i$ choose a point $x_{i} \in \theta_{i}$. Let $x \in C_{\lambda}$. Then $x$ is in $\theta_{i}$ for some $i$. Taylor's Theorem gives, for each $j \in\{1, \cdots, p\}$,

$$
\begin{aligned}
\mid F^{j}\left(x_{i}\right) & -F^{j}(x) \mid \\
\leqq & \sum_{k=1}^{\lambda}(k !)^{-1} \sum_{i_{1}=1}^{m} \cdots \sum_{i_{k}=1}^{m}\left|F_{i_{1} \cdots i_{k}}^{j}(x)\right| \cdot\left|x_{i}^{i_{1}}-x^{i_{1}}\right| \cdots\left|x_{i}^{i_{k}}-x^{i_{k}}\right| \\
& +((\lambda+1) !)^{-1} m^{\lambda+1} M q_{\lambda}^{-\lambda-1} \\
\leqq & \delta_{\lambda} \sum_{k=1}^{2}(k !)^{-1} m^{k} q_{\lambda}^{-k}+((\lambda+1) !)^{-1} m^{\lambda+1} M q_{\lambda}^{-\lambda-1} \\
\leqq & \left(2 q_{\lambda}\right)^{-m / p}\left(\varepsilon^{\prime}\right)^{1 / p} 2^{-2} .
\end{aligned}
$$

Therefore $F\left(C_{\lambda}\right)$ is contained in $\left(q_{\lambda}+1\right)^{m}$ cubes in $R^{p}$ of equal edges: and of total volume not exceeding $\varepsilon^{\prime}$.

(iv) Let $h$ be any natural number such that $0 \leqq h<\lambda$. Let $\left\{\theta_{i}: i=1, \cdots,\left(q_{h}+1\right)^{m}\right\}$ be a family of cubes in $[0,1]^{m}$ of edges $q_{h}^{-1}$, which covers $[0,1]^{m}$. Let $P, Q$ be a partition of $\left\{1, \cdots,\left(q_{h}+1\right)^{m}\right\}$ with the following properties: if $i \in P$ then some $(h+1) s t$ partial derivative of $F$ has absolute value greater than $2^{-2} \delta_{h+1}$ at some point in $\theta_{i}$; if $i \in Q$ then every $(h+1) s t$ partial derivative of $F$ is bounded in absolute value by $2^{-1} \delta_{h+1}$ on $\theta_{i}$. Then clearly $C_{h} \subset \bigcup_{i \in P}\left(\theta_{i} \cap C_{h}\right)$. Let $U_{i}$ be the cube of edge $3 q_{h}^{-1}$ and with the same center as $\theta_{i}$, the edges of $U_{i}$ being parallel to corresponding edges of $\theta_{i}$. Then every $(h+1) s t$ partial derivative of $F$ varies by at most $3 q_{i}^{-1} \mathrm{mM}$, or less than $2^{-3} \delta_{h+1}$, on $U_{i}$. Thus $i \in P$ implies some $(h+1) s t$ partial derivative has absolute value greater than $2^{-3} \delta_{h+1}$ on $U_{i}$. But $\left\langle m, p, \theta_{i}, q_{h}^{-1}\right.$, $F, M\rangle$. Therefore, by the definitions of $n_{h}$ and $\delta_{h}$ and by 2.2 and 2.3 , the set $F\left(C_{h}\right)$ is contained in $\left(q_{h}+1\right)^{m} n_{h}$ cubes of equal edges. and of total volume not exceeding $\left(q_{h}+1\right)^{m} \varepsilon_{h}$ which is less than $\varepsilon^{\prime}$.

(v) Combining (ii), (iii), and (iv) we see that $\left\{F(x): x \in K, J_{F}(x) \leqq \nu\right\}$ 
is contained in a union of $\left(q_{\lambda}+1\right)^{m}$ cubes of equal edges of total volume not exceeding $\varepsilon^{\prime}$, and of $\left(q_{h}+1\right)^{m} n_{h}$ cubes of equal edges of total volume not exceeding $\varepsilon^{\prime}(0 \leqq h<\lambda)$. Therefore, by definition of $n$, the set $\left\{F(x): x \in K, J_{F}(x) \leqq \nu\right\}$ is contained in a union of $n$ cubes in $R^{p}$, whose edges are equal and whose total volume does not exceed $2^{p+1}(\lambda+1) \varepsilon^{\prime}$ which is less than $\varepsilon$.

In 2.1 the mapping is assumed to be $2+2^{-1}(m-p)(m-p+1)$ times continuously differentiable. The classical theorem ([4], [5]) assumes it is only $m-p+1$ times continuously differentiable if $m \geqq p$ (while, of course, no differentiability is needed if $m<p$ ). The author has not been able to obtain a constructive proof without assuming higher differentiability than $m-p+1$.

\section{REFERENCES}

1. E. Bishop, Foundations of Constructive Analysis, McGraw Hill, Inc., New York, 1967.

2. J. Milnor, Topology from the Differential Viewpoint, University Press of Virginia, Charlottesville, 1965.

3. L. S. Pontryagin, Smooth manifolds and their applications in homotopy theory, Amer. Math. Soc. Translations (2) 11 (1959), 1-114; translated from Trudy Inst. Steklov 45 (1955).

4. A. Sard, The measure of the critical points of differentiable maps, Bull. Amer. Math. Soc. 48 (1942), 883-890.

5. A. Ya. Dubovickii, On differentiable mappings of an n-dimensional cube into a k-dimensional cube, Mat. Sbornik N.S. (74) 32 (1953), 443-464 (in Russian).

Received March 9, 1970. The research leading to this paper was partially supported by NSF Grant GP-8040.

University of California, SAN Diego 



\section{PACIFIC JOURNAL OF MATHEMATICS}

\section{EDITORS}

H. SAMELson

Stanford University

Stanford, California 94305

R. R. PHelPS

University of Washington

Seattle, Washington 98105
J. Dugundu

Department of Mathematics

University of Southern Californle

Los Angeles, California 9.0007

RICHARD ARENS

University of California

Los Angeles, California 9.0024

\section{ASSOCIATE EDITORS}
E. F. BECKENBACH
B. H. NeumanN
F. WOLE
K. Yoshida

\section{SUPPORTING INSTITUTIONS}

UNIVERSITY OF BRITISH COLUMBIA

CALIFORNIA INSTITUTE OF TECHNOLOGY

UNIVERSITY OF CALIFORNIA

MONTANA STATE UNIVERSITY

UNIVERSITY OF NEVADA

NEW MEXICO STATE UNIVERSITY

OREGON STATE UNIVERSITY

UNIVERSITY OF OREGON

OSAKA UNIVERSITY

UNIVERSITY OF SOUTHERN CALIFORNIA

\author{
STANFORD UNIVERSITY \\ UNIVERSITY OF TOKYO \\ UNIVERSITY OF UTAH \\ WASHINGTON STATE UNIVERSITY \\ UNIVERSITY OF WASHINGTON \\ AMERICAN MATHEMATICAL SOCIETY \\ CHEVRON RESEARCH CORPORATION \\ NAVAL WEAPONS CENTER
}

The Supporting Institutions listed above contribute to the cost of publication of this Journal, but they are not owners or publishers and have no responsibility for its content or policies.

Mathematical papers intended for publication in the Pacific Journal of Mathematics should be in typed form or offset-reproduced, (not dittoed), double spaced with large margins. Underline Greek letters in red, German in green, and script in blue. The first paragraph or two must be capable of being used separately as a synopsis of the entire paper. The editorial "we" must not be used in the synopsis, and items of the bibliography should not be cited there unless absolutely necessary, in which case they must be identified by author and Journal, rather than by item number. Manuscripts, in duplicate if possible, may be sent to any one of the four editors. Please classify according to the scheme of Math. Rev. Index to Vol. 39. All other communications to the editors should be addressed to the managing editor, Richard Arens, University of California, Los Angeles, California, 90024.

50 reprints are provided free for each article; additional copies may be obtained at cost in multiples of 50 .

The Pacific Journal of Mathematics is published monthly. Effective with Volume 16 the price per volume (3 numbers) is $\$ 8.00$; single issues, $\$ 3.00$. Special price for current issues to individual faculty members of supporting institutions and to individual members of the American Mathematical Society: $\$ 4.00$ per volume; single issues $\$ 1.50$. Back numbers are available.

Subscriptions, orders for back numbers, and changes of address should be sent to Pacific Journal of Mathematics, 103 Highland Boulevard, Berkeley, California, 94708.

PUBLISHED BY PACIFIC JOURNAL OF MATHEMATICS, A NON-PROFIT CORPORATION

Printed at Kokusai Bunken Insatsusha (International Academic Printing Co., Ltd.), 7-17, Fujimi 2-chome, Chiyoda-ku, Tokyo, Japan. 


\section{Pacific Journal of Mathematics}

\section{Vol. 36, No. 2 December, 1971}

George E. Andrews, On a partition problem of H. L. Alder ............ 279

Thomas Craig Brown, An interesting combinatorial method in the theory of locally finite semigroups .......................... 285

Yuen-Kwok Chan, A constructive proof of Sard's theorem ............. 291

Charles Vernon Coffman, Spectral theory of monotone Hammerstein

operators...................................... 303

Edward Dewey Davis, Regular sequences and minimal bases .......... 323

Israel (Yitzchak) Nathan Herstein and Lance W. Small, Regular elements in

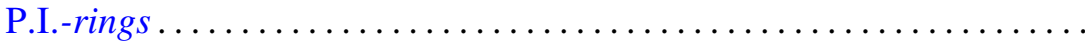

Marcel Herzog, Intersections of nilpotent Hall subgroups ..............

W. N. Hudson, Volterra transformations of the Wiener measure on the space

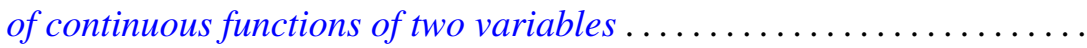

J. H. V. Hunt, An n-arc theorem for Peano spaces ................ 351

Arnold Joseph Insel, A decomposition theorem for topological group

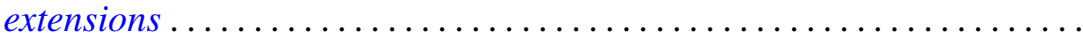

Caulton Lee Irwin, Inverting operators for singular boundary value

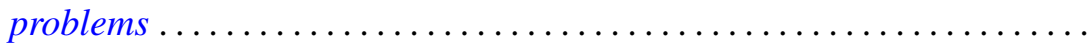

Abraham A. Klein, Matrix rings of finite degree of nilpotency ............ 387

Wei-Eihn Kuan, On the hyperplane section through a rational point of an algebraic variety...

John Hathway Lindsey, II, On a six-dimensional projective representation of $\mathrm{PSU}_{4}(3)$

Jorge Martinez, Approximation by archimedean lattice cones ...

J. F. McClendon, On stable fiber space obstructions .........

Mitsuru Nakai and Leo Sario, Behavior of Green lines at the Kuramochi boundary of a Riemann surface ....................

Donald Steven Passman, Linear identities in group rings. I. .

Donald Steven Passman, Linear identities in group rings. II ...

David S. Promislow, The Kakutani theorem for tensor products of

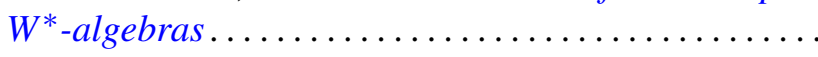

Richard Lewis Roth, On the conjugating representation of a finite group

Bert Alan Taylor, On weighted polynomial approximation of entire functions...

William Charles Waterhouse, Divisor classes in pseudo Galois

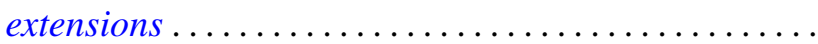

Chi Song Wong, Subadditive functions ...

Ta-Sun $\mathrm{Wu}$, A note on the minimality of certain bitransformation groups 